PROCEEDINGS OF THE

AMERICAN MATHEMATICAL SOCIETY

Volume 124, Number 12, December 1996, Pages 3753-3754

S 0002-9939(96)03525-3

\title{
NOTE ON THE BRADLEY AND RAMANUJAN SUMMATION
}

\author{
CHU WENCHANG
}

(Communicated by J. Marshall Ash)

\begin{abstract}
The hypergeometric series of Bradley and Ramanujan is evaluated by means of the binomial convolutions of Hagen and Rothe, which presents, alternatively, a short proof of the recent result of Bradley about Ramanujan's enigmatic claim.
\end{abstract}

For complex numbers $\alpha, \beta, \gamma$ and integer $\delta$, define the sum of Ramanujan type by

$$
S_{\delta}(\alpha, \beta, \gamma ; z)=\gamma \sum_{k=0}^{\infty} \frac{(\alpha)_{k}}{k !} \frac{\Gamma(\beta+z k) \Gamma(k+\gamma+z k)}{\Gamma(\delta+k+\alpha+\beta+z k) \Gamma(1+\gamma+z k)} .
$$

It reduces, under parameter replacements $\delta \rightarrow 0, \beta \rightarrow 1+\beta$ and $\gamma \rightarrow m$, to the sum of Bradley [2], who has recently presented a most plausible interpretation for Ramanujan's enigmatic claim, which may be restated in terms of $S$-sum as "the difference between $\Gamma(1+\beta-m) / \Gamma(1+\alpha+\beta-m)$ and $S_{0}(\alpha, 1+\beta, m ; z) \quad \ldots$ " (cf. Bradley [2]).

Theorem. With the $S$-function defined as above, we have the following evaluations:

A: Bradley $[2,1994]$. For $\operatorname{Re}(\delta+\beta-\gamma)>0$,

$$
S_{\delta}(\alpha, \beta, \gamma ; 0)=\frac{\Gamma(\beta) \Gamma(\delta+\beta-\gamma)}{\Gamma(\delta+\beta) \Gamma(\delta+\alpha+\beta-\gamma)} .
$$

B. For $\operatorname{Re}(1-\alpha-\beta+\gamma)>0$,

$$
S_{\delta}(\alpha, \beta, \gamma ;-1)=\frac{\Gamma(\beta) \Gamma(1-\beta) \Gamma(1-\alpha-\beta+\gamma)}{\Gamma(\delta+\alpha+\beta) \Gamma(1-\alpha-\beta) \Gamma(1-\beta+\gamma)} .
$$

C: Bradley $[2,1994]$. When $\alpha$ is a non-positive integer,

$$
S_{0}(\alpha, \beta, \gamma ; z)=\frac{\Gamma(\beta-\gamma)}{\Gamma(\alpha+\beta-\gamma)}
$$

D. When $\alpha$ is a non-positive integer,

$$
S_{1}(\alpha, \beta, \gamma ; z)=\frac{\alpha z-\beta+\gamma}{\alpha z-\beta} \frac{\Gamma(\beta-\gamma)}{\Gamma(1+\alpha+\beta-\gamma)} .
$$

Received by the editors January 3, 1995 and, in revised form, May 23, 1995.

1991 Mathematics Subject Classification. Primary 33A30; Secondary 05A19.

Key words and phrases. Binomial convolution, Hypergeometric series, The Gauss summation theorem.

The author was partially supported by IAMI (CNR, Milano), 1994. 
Proof. For $z=0$ and -1 , we can rewrite

$$
\begin{aligned}
S_{\delta}(\alpha, \beta, \gamma ; 0) & =\frac{\Gamma(\beta)}{\Gamma(\delta+\alpha+\beta)} \times{ }_{2} F_{1}\left[\begin{array}{c}
\alpha, \gamma \\
\delta+\alpha+\beta
\end{array}\right], \\
S_{\delta}(\alpha, \beta, \gamma ;-1) & =\frac{\Gamma(\beta)}{\Gamma(\delta+\alpha+\beta)} \times{ }_{2} F_{1}\left[\begin{array}{c}
\alpha,-\gamma \\
1-\beta
\end{array}\right],
\end{aligned}
$$

which yield (2a) and (2b), respectively, in view of the Gauss theorem [1] (see also [3])

$$
{ }_{2} F_{1}\left[\begin{array}{cc}
a, & b \\
& c
\end{array}\right]=\frac{\Gamma(c-a) \Gamma(c-b)}{\Gamma(c) \Gamma(c-a-b)}, \quad \operatorname{Re}(c-a-b)>0
$$

When $\alpha=-n$, a non-positive integer, the $S$-function defined in (1) may be reformulated as

$$
\begin{aligned}
S_{\delta}(-n, \beta, \gamma ; z) & =\gamma \sum_{k=0}^{n}(-1)^{\delta+n}\left(\begin{array}{c}
n \\
k
\end{array}\right) \frac{(\gamma+z k)_{k}}{\gamma+z k}(1-\beta-z k)_{n-k-\delta} \\
& =\sum_{k=0}^{n} \frac{\gamma}{\gamma+z k}\left(\begin{array}{c}
-\gamma-z k \\
k
\end{array}\right) \frac{n !}{(\beta+z k)_{\delta}}\left(\begin{array}{c}
\delta-1+\beta+z k \\
n-k
\end{array}\right),
\end{aligned}
$$

which reduce, respectively for $\delta=0$ and 1 , to

$$
\begin{aligned}
& S_{0}(-n, \beta, \gamma ; z)=n !\left(\begin{array}{c}
\beta-\gamma-1 \\
n
\end{array}\right), \\
& S_{1}(-n, \beta, \gamma ; z)=\frac{n !}{\beta+z n} \frac{\beta-\gamma+z n}{\beta-\gamma}\left(\begin{array}{c}
\beta-\gamma \\
n
\end{array}\right),
\end{aligned}
$$

by means of the Hagen-Rothe [5] (see also $[3,4]$ ) formulae

$$
\sum_{k=0}^{n} \frac{a}{a+b k}\left(\begin{array}{c}
a+b k \\
k
\end{array}\right)\left(\begin{array}{c}
c-b k \\
n-k
\end{array}\right)=\left(\begin{array}{c}
a+c \\
n
\end{array}\right),
$$

(5b) $\sum_{k=0}^{n} \frac{a}{a+b k}\left(\begin{array}{c}a+b k \\ k\end{array}\right) \frac{c-b n}{c-b k}\left(\begin{array}{c}c-b k \\ n-k\end{array}\right)=\frac{a+c-b n}{a+c}\left(\begin{array}{c}a+c \\ n\end{array}\right)$.

It is obvious that (2c) and (2d) are respectively the reformulations of (4a) and (4b).

\section{REFERENCES}

1. W. N. Bailey, Generalized Hypergeometric Series, Cambridge University Press, Cambridge, 1935.

2. D. Bradley, On a claim of Ramanujan about certian hypergeometric series, Proc. Amer. Math. Soc.121:4 (1994), 1145-1149. MR 94j:33003

3. W. Ch. Chu, Inversion techniques and combinatorial identities, Boll. UMI (7) 7-B (1993), 737-760. MR 95e:33006

4. W. Ch. Chu \& L. C. Hsu, Some new applications of Gould-Hsu inversions, J. Combin. Informat. \& System Science 14:1 (1990), 1-4. MR 92c:05018

5. H. W. Gould, Some generalizations of Vandermonde's convolution, Amer. Math. Month.63:1 (1956), 84-91. MR 17:702g

Istituto di Matematica, "Guido Castelnuovo", Università degli Studi di Roma "LA SAPIENZA", Roma, ItALia

E-mail address: WENCHANG@mat.uniroma1.it 